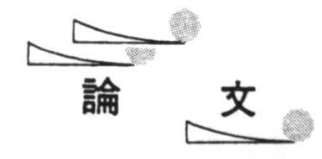

\title{
Configuration and Frequency of Longitudinal Vortices Shedding from Two Circular Cylinders in Cruciform Arrangement*
}

\author{
Tsutomu TAKAhashI $^{* *}$, László BARANYI ${ }^{* * *}$ and Masataka ShIRAKASHI ${ }^{* *}$
}

\begin{abstract}
A new vortex excitation of a circular cylinder placed in an otherwise uniform flow was reported in earlier papers by the present authors. This excitation is induced by longitudinal vortices which are originated by setting another cylinder downstream of the first one in cruciform arrangement with a gap less than one half of the cylinder diameter. In this work, the configuration and shedding frequency of the longitudinal vortices causing the excitation were investigated through flow visualization experiments in a water tunnel and quantitative measurements in a wind tunnel. The visualized configurations of the two types of longitudinal vortices, the trailing vortex and the necklace vortex, correlated well with their corresponding contours obtained from pressure-velocity cross-spectrum measurements in the wind tunnel. Strouhal numbers of the longitudinal vortices were also investigated over a wide range of the Reynolds number $R e$, by using both a wind tunnel and a water tunnel. While the Strouhal numbers for the two longitudinal vortices are practically independent of $R e$ for $R e>10000$, they increase appreciably with an increasing Reynolds number in the range of $R e<10000$.
\end{abstract}

Key words : Two circular cylinders, Cruciform arrangement, Vortex excitation, Longitudinal Vortex, Pressure-velocity cross-spectrum, Strouhal number

\section{Introduction}

The interference effect of neighboring bodies on the oscillation of an elastically supported cylindrical body placed in a cross flow is of practical importance because it can lead to undesirable oscillations ${ }^{1 /-3)}$. Compared with two-dimensional arrangements, reports concerning the flow around two cylinders in a cruciform arrangement as shown in Figure 1 are rather few in numbers in spite of the fact that there are many equipment available in laboratories and plants with such a cylinder arrangement, e.g., array of pipes in heat exchangers, turbulence generating grids in wind tunnels, etc.

In earlier papers ${ }^{4), 5)}$ the present authors reported that large cross-flow oscillations such as shown in Fig-

* Received on January 21, 1999

** A regular member, Nagaoka University of Technology, Department of Mechanical Engineering

*** University of Miskolc, Faculty of Mechanical Engineering

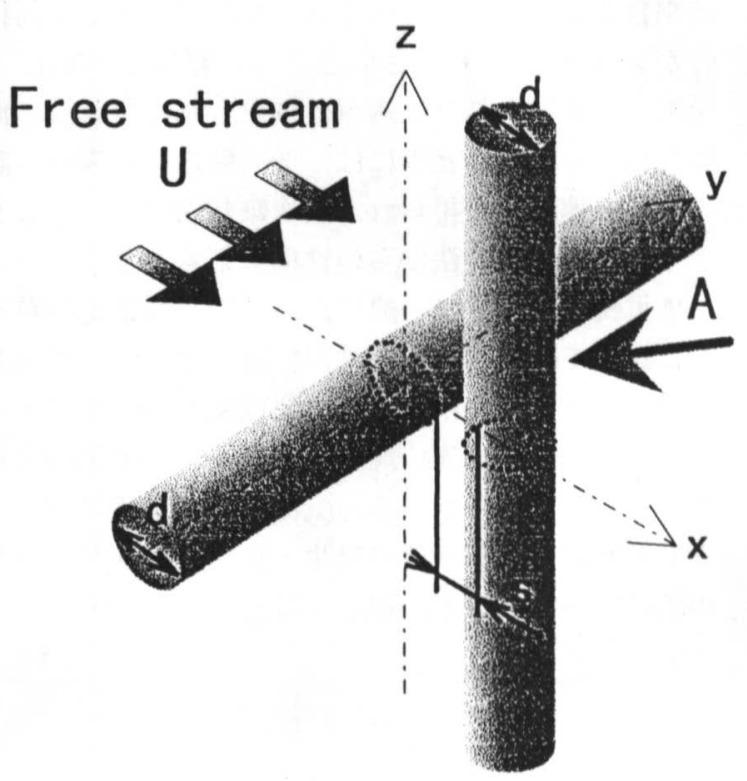

Fig. 1 Cylinder Arrangement and Coordinate System. 
ure 2 can be induced on an elastically supported circular cylinder when another cylinder is set downstream in the cruciform arrangement. A large excitation peak can be seen in Figure $2(\mathrm{a})$ at the free stream velocity $U=8 \mathrm{~m} / \mathrm{s}$ when the gap-to-diameter ratio is $s / d=$ 0.125 . As can be seen in this figure, the Karman vortex excitation, characteristic for the single cylinder at $U=3 \mathrm{~m} / \mathrm{s}$, is completely depressed ${ }^{6)}$. When the gap $\dot{s}$ between the two cylinders was varied while the free stream velocity $U$ was fixed at $8 \mathrm{~m} / \mathrm{s}$, a new oscillation appeared in the range $s / d \leq 0.25$. Another new oscillation peak appeared in $0.25 \leq s / d \leq 0.5$ while $U=14$ $\mathrm{m} / \mathrm{s}$. These peaks can be seen in Figures $2(\mathrm{~b})$ and (c), respectively. Measurements on the fluctuating pressure distribution on the upstream cylinder surface near the crossing were curried out with a fixed system in a wind tunnel. The results showed that there exists a dominant frequency component in the pressure fluctuation that can make the upstream cylinder resonate if it is supported elastically. To facilitate the evaluation
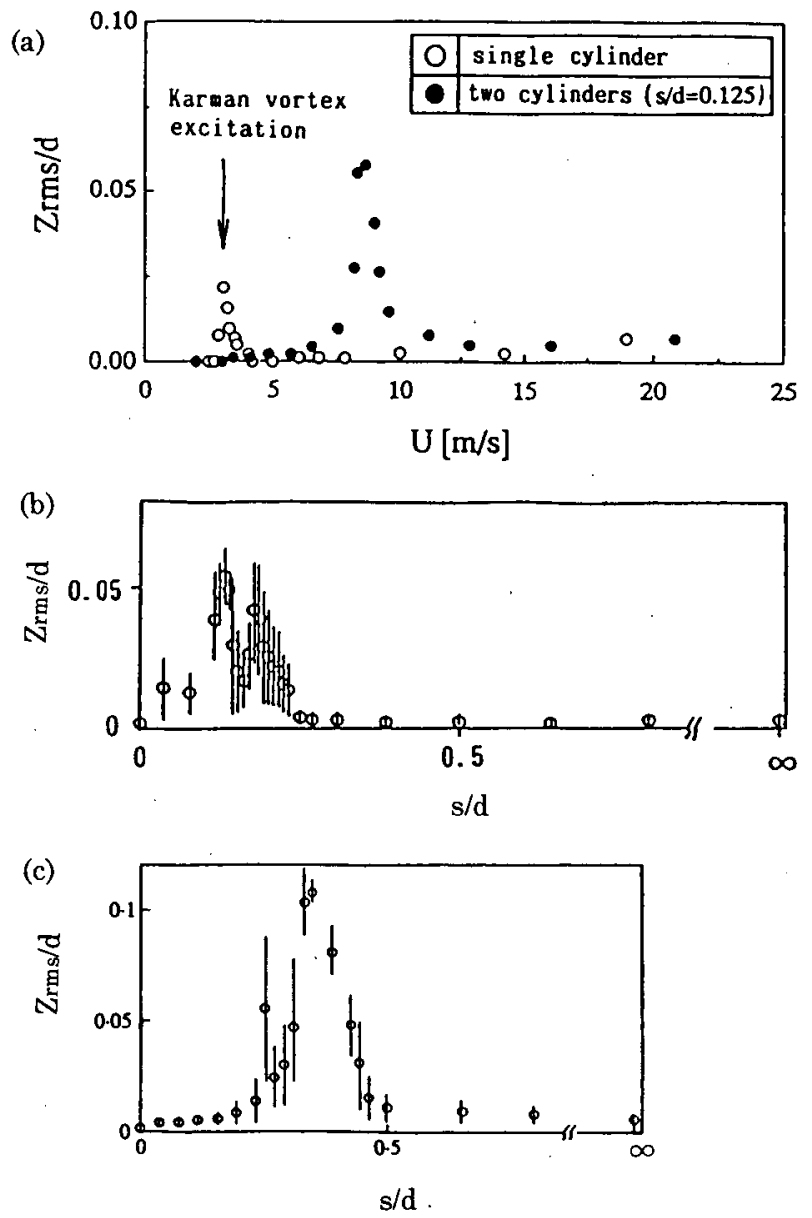

Fig. 2 Longitudinal Vortex Excitation; The Cylinder Diameter $d=26 \mathrm{~mm}$ and the Natural Frequency of Upstream Cylinder is $24 \mathrm{~Hz}$. (Zrms : rms value of $z$ Displacement of the Upstream Cylinder) of the excitation lift force, pressure fluctuation at a point on the upstream cylinder was selected as the reference signal for the phase averaging method because the dominant frequency component in the pressure was most clearly detected at that position. Based on the phase-average pressure fluctuation distribution maps obtained in this way and observations of flow visualized by smoke wire method, further comparing them with those of other researchers also for fixed systems ${ }^{7)-9}$, we conclude that the large oscillation of the upstream cylinder is caused by the periodic shedding of either the trailing vortices or necklace vortices as illustrated in Figure 3. Since both of these vortices have axes parallel to the free stream we called the new oscillation "longitudinal vortex excitation". It was shown that the formation of these longitudinal vortices depends strongly on $s / d$, and that the Strouhal number of these vortices are practically independent of the Reynolds number $R e$ in the range of $R e$ attained by wind tunnel experiments. It was found that trailing vortices shed when $0 \leq s / d \leq 0.25$ (Region A in Figure 4) with Strouhal numbers ranging from 0.06 to 0.09 and the necklace vortices shed with a Strouhal number around 0.04 when $0.25 \leq s / d \leq 0.5$ (Region $\mathrm{B}$ in Figure 4).

The illustrations of the longitudinal vortices in Figure 3 are rather rude since they were obtained from indirect measurements such as those of the pressure on the upstream cylinder surface, compared with observations in flow-visualization experiments carried out separately on a fixed system. Besides, it is natural to expect that the Strouhal number of such vortex shedding will depend on the Reynolds number $R e$ when the latter is not high enough. Therefore, the specific aim of this work is to investigate the structure of the vortices which are responsible for the longitudinal vortex excitation, based on direct observation supported by quantitative measurements, and to clarify the dependence of Strouhal number of the longitudinal vortices on $R e$ over a wide range of $R e$ including lower values of $R e$ reported so far by carrying out experiments both in a water tunnel and a wind tunnel.

\section{Experimental apparatus and procedure}

A wind tunnel was used to carry out quantitative measurements of velocity field around the crossing. An outline of the apparatus for wind tunnel experiments and the coordinate system used in this paper are shown in Figure 5. The wind tunnel is a blow down type. The maximum attainable velocity is $25 \mathrm{~m} / \mathrm{s}$ and 


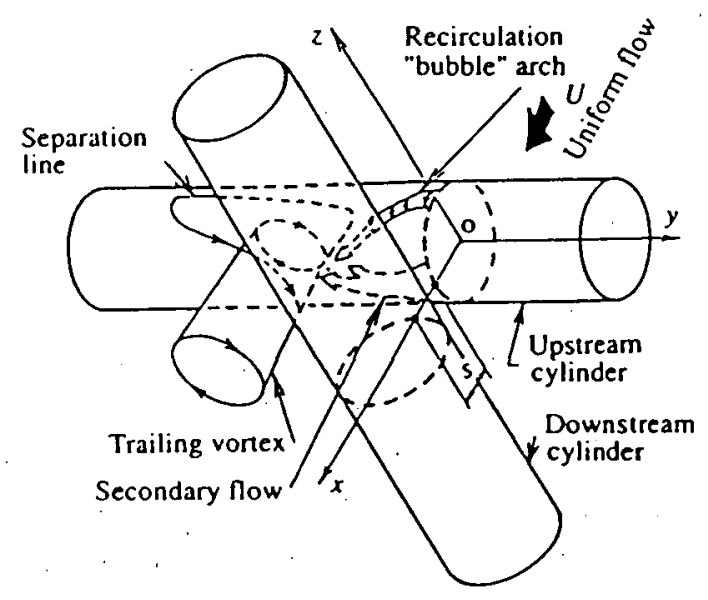

(a)Trailing Vortex: Region A $(s / d \leq 0.25)$

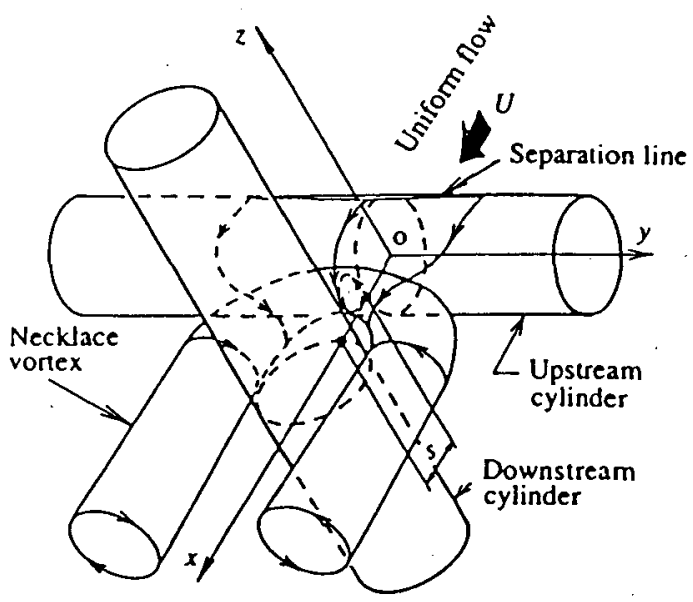

(b) Necklace Vortex: Region B $(0.25 \leq s / d \leq 0.5)$

Fig. 3 Flow Structure near the Crossing.

the turbulence intensity is less than $0.4 \%$. The measuring channel is $320 \mathrm{~mm} \times 320 \mathrm{~mm}$ in cross section and $1,000 \mathrm{~mm}$ in length. The upstream cylinder with a diameter of $26 \mathrm{~mm}$ is fixed horizontally in the central plane of the measuring section. There is a pressure hole of $0.4 \mathrm{~mm}$ in diameter on the upstream cylinder surface and it can use to measure the difference of the pressure on the cylinder surface and the static pressure in the free stream. The position of the pressure hole is set at the location where the pressure fluctuation due to the longitudinal vortex shedding is most clearly detected, i.e. the angle $\alpha$ from the stagnation line is 60 degrees and $y / d=1.0^{5}$. In that paper, the pressure detected at this position was used as a reference signal to the fluctuating lift force causing the longitudinal excitation. The tubing connecting the pressure tap to the transducer is short enough to allow detecting pressure fluctuation with frequencies up to the maximum shedding frequency of Kármán vortices measured in this experiment. The vertical downstream

\begin{tabular}{|l|c|c|c|}
\cline { 2 - 4 } \multicolumn{1}{c|}{} & \multicolumn{1}{c|}{$\begin{array}{c}\text { Fixed } \\
\text { system }\end{array}$} & $\begin{array}{c}\text { Elastic } \\
\text { system }\end{array}$ & $R e$ \\
\hline $\begin{array}{l}\text { by fluctuating } \\
\text { velocity[5] }\end{array}$ & - & $-\cdot-\cdot-$ & 13700 \\
\hline & - & $----\cdot-$ & 24000 \\
\hline $\begin{array}{l}\text { by fluctuating } \\
\text { pressure[5] }\end{array}$ & + & & $\begin{array}{c}11500 \\
-30000\end{array}$ \\
\hline $\begin{array}{l}\text { by flow } \\
\text { visualization[5] }\end{array}$ & $\square$ & & 3400 \\
\hline Yamada et.al.[11] & $\square$ & & $\begin{array}{c}20000 \\
\sim 30000\end{array}$ \\
\hline Tomita et.al. [12] & $\square$ & & 8000 \\
\hline
\end{tabular}

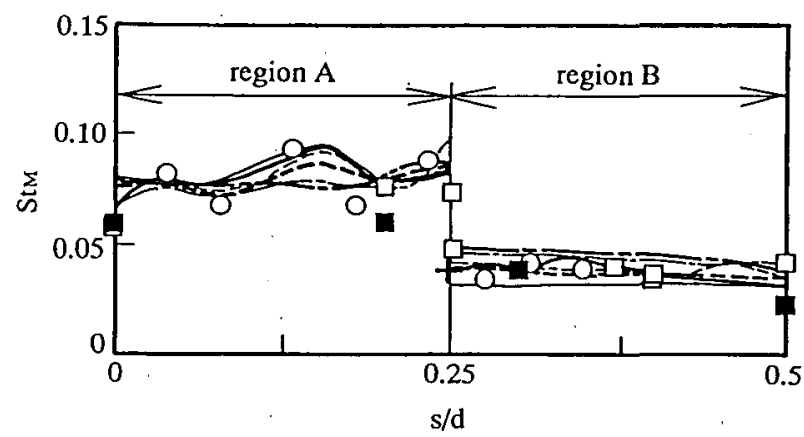

Fig. 4 Relationship between the Strouhal Number for the Longitudinal Vortex Shedding and the Gap-to Diameter Ratio Reported so far.

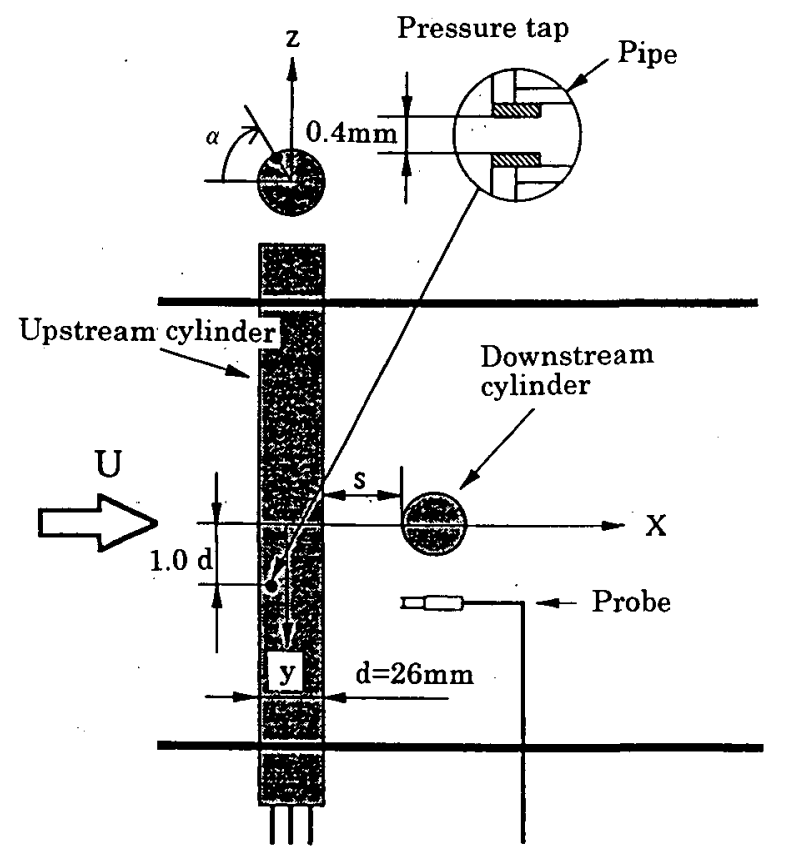

Fig. 5 Outline of the Apparatus of the Wind Tunnel Experiment (Plan view).

cylinder, the diameter of which is equal to that of the upstream one, is mounted on a traversing device that can adjust the gap between cylinders with an accuracy of $0.05 \mathrm{~mm}$. The free stream velocity $U$ is measured by a Pitot tube for $U \geq 8 \mathrm{~m} / \mathrm{s}$, and by a ring type Kármán vortex velocimeter for $U<8 \mathrm{~m} / \mathrm{s}$. Hot wire anemometry was applied to measure the fluctuating $x$ component $u$ of the velocity. 
A water tunnel with a square test section of $200 \mathrm{~mm}$ and a length of $550 \mathrm{~mm}$ is used for visualization experiments and measurements in the low Reynolds number range, say $R e<5100$. The diameters of the cylinders are $10,13,18$ or $22 \mathrm{~mm}$ depending on the range of $R e$ to be measured. For flow visualization, dye streaks are introduced to the streamlines leading to the upstream stagnation points of the upstream cylinder. A hot film probe is used to measure $u$, the $x$-component of the velocity fluctuation.

\section{Results and discussion}

3.1 Reference pressure and velocity fluctuation around the crossing

Examples of fluctuating pressure $p$ at the reference point of the upstream cylinder surface are presented in Figure 6. In this figure, the gap $s$ is varied while the free stream velocity $U$ is kept at a constant value of 8 $\mathrm{m} / \mathrm{s}$. When the gap-to-diameter ratio $s / d=0.08$, a dominant frequency component is observed in the fluctuating reference pressure $p$, and the Strouhal number for the frequency correlates well with those of the trailing vortices in Region $\mathrm{A}$ in Figure 4 . When $s / d=$ 0.28 , the frequency of the dominant component in the pressure signal decreases to a value which corresponds to the Strouhal number of around 0.04, i.e. that of the necklace vortex. It is assured from these results that the reference pressure $p$ represents the fluctuations caused by the periodic shedding of the longitudinal vortices of two types. The transition from the trailing vortex regime to the necklace vortex regime occurs abruptly at $s / d=0.25$, where the two frequency components appear alternately as seen in Figure 6.

- A similar phenomenon is observed in the $x$ component of the velocity fluctuation $u$, at a point near the crossing of the two cylinders, as shown in Figure 7 , although the velocity signals are blurred by high frequency 'noise' due to turbulence. The $u$ signals in Figure 7 were detected by a hot wire probe set at the position where the dominant components with frequencies equal to those in $p$ were most clearly discerned. When the probe was shifted to another position, the dominant components became less discernible.

\section{2 Cross-spectrum between the reference pressure} and velocities near the crossing

The similar behaviors of the dominant components in the reference pressure $p$ and the velocity $u$ at a point near the crossing infers that they are caused by the same origin, i.e. periodic shedding of identical longitudinal vortices. When a longitudinal vortex sheds
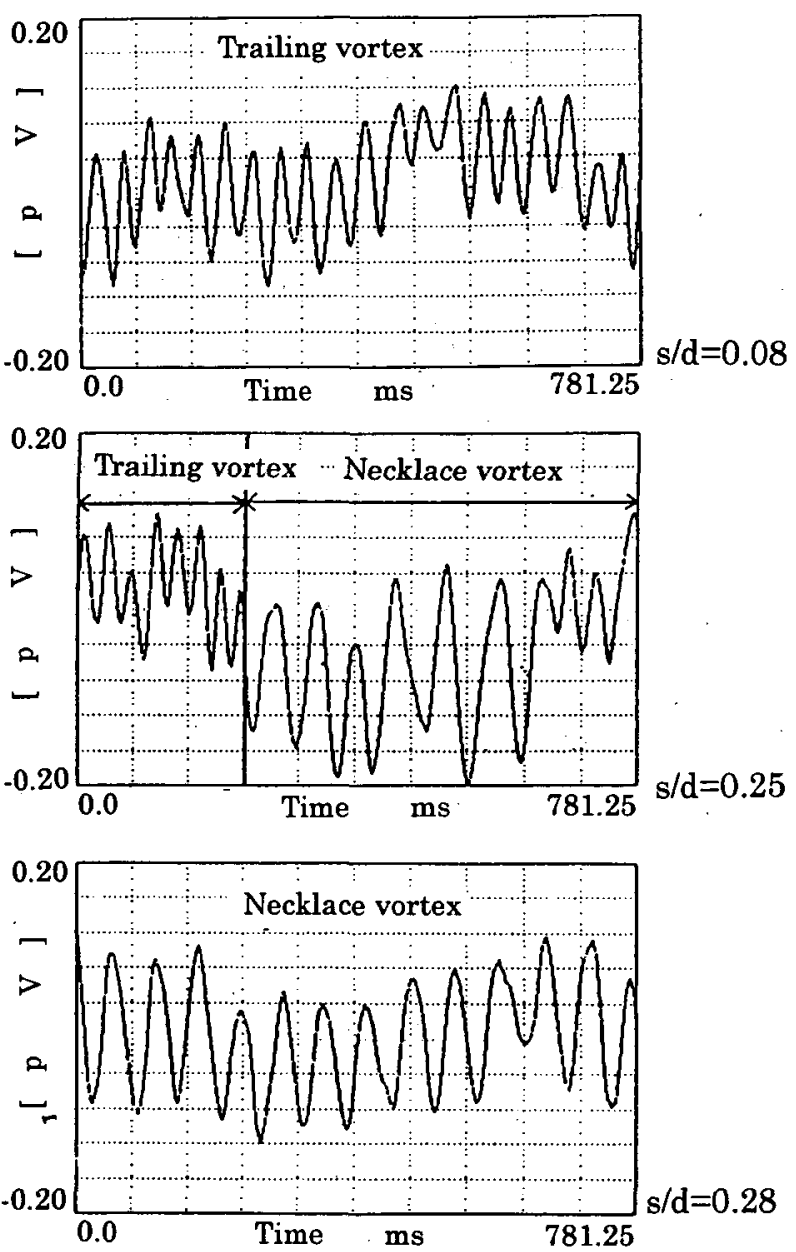

Fig. 6 Oscillograms of the Reference Pressure on the Upstream Cylinder Surface $(U=8 \mathrm{~m} / \mathrm{s}, R e=13,900$, $y / d=1.0, \alpha=60$ degrees). Vortex Types Shown in Figures were Inferred from Comparison between a Domain Frequency of the Fluctuation and $S t_{M}$ in Figure 4.

from the upstream cylinder, it causes a change of pressure acting on the upstream cylinder, and causes a change of velocity $u$ in the wake where the vortex is formed. There is a phase shift between the two signals; the change in pressure $p$ may precede that of in velocity $u$. To investigate this coherent periodic structure of the flow field which induces the excitation of the upstream cylinder, the hot wire probe was scanned over a range around the crossing to measure $u$, while the pressure $p$ at the reference point on the upstream cylinder was detected simultaneously. Crossspectrum $S_{p u}$ between $p$ and $u$ was obtained to extract dominant frequency components in $p$ and $u$ which are synchronized with each other. Cross-spectrum $S_{p u}$ for signals $p$ and $u$ in Figures 6 and 7, are shown in Figure 8. A very sharp peak appears in $S_{p u}$ for $s / d=0.08$ and another one for $s / d=0.28$, the frequencies of 

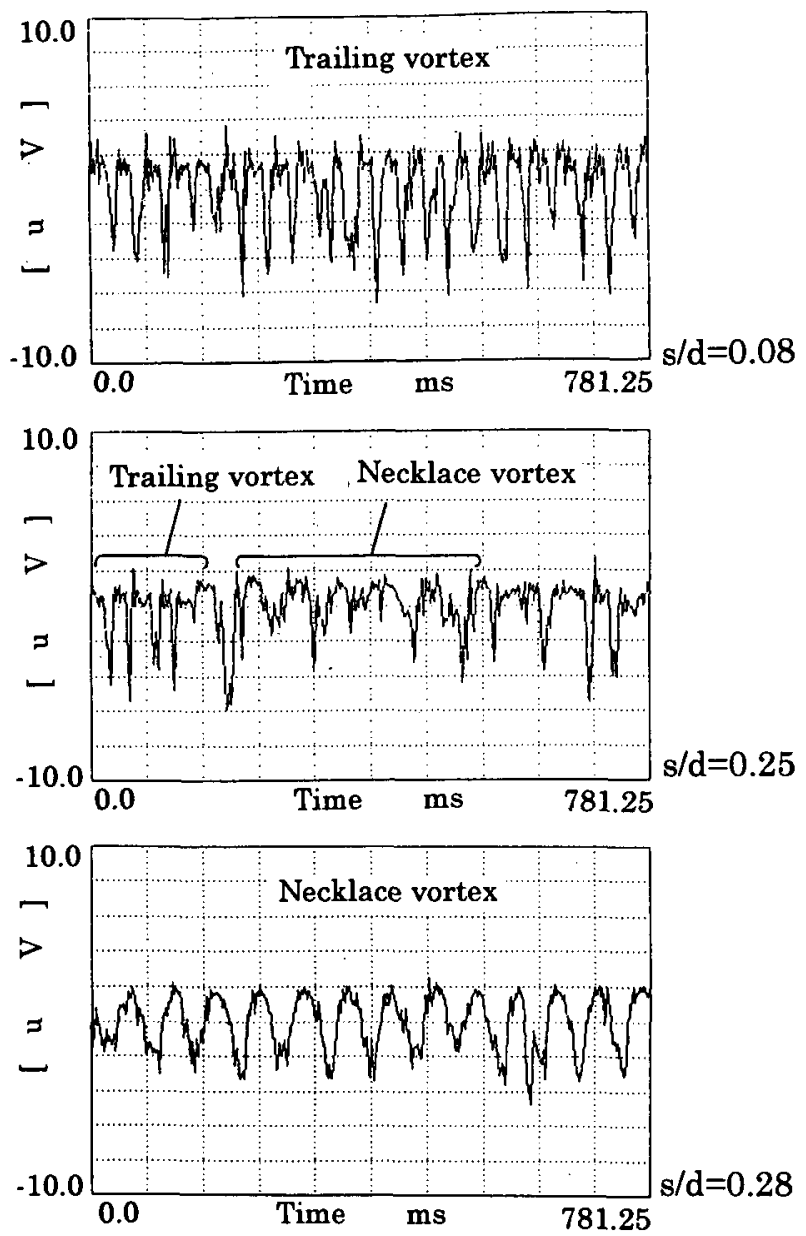

Fig. 7 Oscillograms of $x$-velocity Component at a Point near the Crossing $(U=8 \mathrm{~m} / \mathrm{s}, R e=13,900, x / d=$ 2. $0, y / d=1.5, z / d=1.0$ ). Vortex Types shown in figures were Inferred from Comparison between a Domain Frequency of the Fluctuation and $S t_{M}$ in Figure 4.

which coincide with the dominant frequency components observed in the oscillograms of pressure $p$ and velocity $u$ in Figures 6 and 7 . The peak in the crossspectrum $S_{p u}$ at a frequency $f_{v M}$ means that both $u$ and $p$ have the same dominant frequencies, i.e. $f_{v M}$. This result confirms that the dominant component in the fluctuating velocity at a point near the crossing is directly connected with that of the reference pressure on the upstream cylinder surface, i.e. both are originated from the same vortex. Information on the phase difference between $p$ and $u$ is missing in $S_{p u}$ and the phase shift of dominant component in $u$ is supposed to depend on the position of the hot wire probe. Two peaks appear in the spectrum for $s / d=0.25$, showing that the two types of longitudinal vortices shed alternately at this gap value.

The frequency, $f_{v M}$ for the peak of the cross-
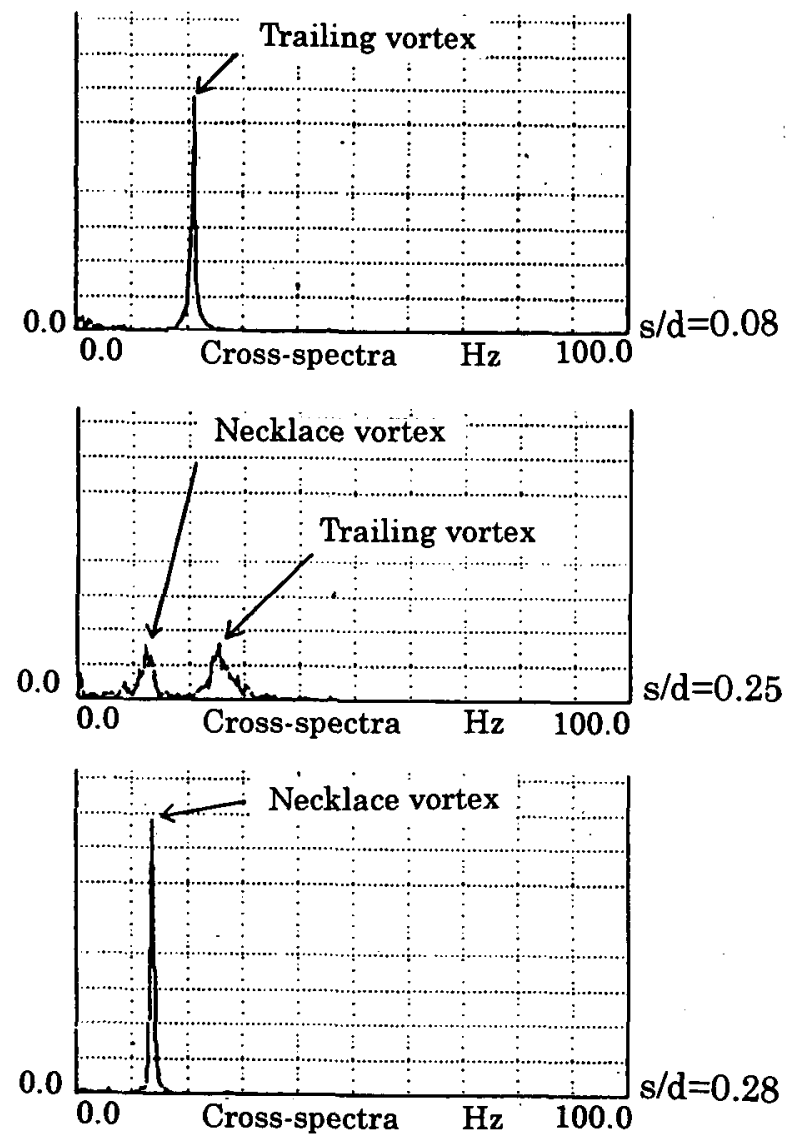

Fig. 8 Cross-spectra of Pressure and Velocity Signals as presented in Figures 6 and 7, respectively.

spectrum $S_{p u}$ was obtained for variable gap. It is reduced to non-dimensional Strouhal number $S t_{M}=f_{v M} d / U$ and plotted against $s / d$ in Figure 9. The measurements were carried out twice, and it is found that the repeatability is excellent even in the region of trailing vortex shedding (Region A in the Figure) where the Strouhal number $S t_{M}$ varies irregularly over a range of $0.05-0.085 . S t_{M}$ is stable and varies slightly around the value of 0.04 over the region of $0.25 \leq s / d \leq 0.5$ where necklace vortices are shed (Region B). In this region, the results of the two test series practically coincide for all $s / d$ values. The cross-hatched solid lines in Figure 9 give the range of Strouhal numbers obtained partly during our previous works for both fixed systems and resonant elastic systems $\mathrm{s}^{4,5)}$, and partly reported by other authors for fixed systems ${ }^{8 / 11}$. Results shown in Figures 8 and 9 verify that the dominant frequency components in the reference pressure $p$, which represents the fluctuating lift force acting on the upstream cylinder, and in the velocity $u$ at a point around the crossing are really caused by the same periodic change of the flow structure inherent to the fixed cruciform two-cylinder configuration, i.e. the peri- 


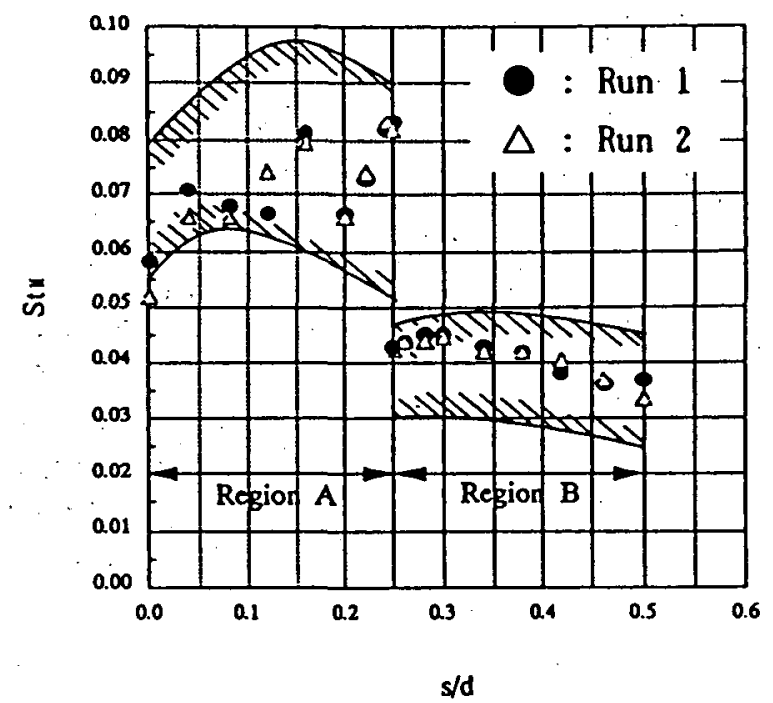

Fig. 9 Strouhal Number for the Longitudinal Vortices obtained from Cross-spectrum. The Experimental Conditions are Identical with those for Figure 8. The Cross-hatched Lines indicate the Region of Strouhal Numbers reported so far.

odic shedding of the longitudinal vortices.

The magnitude of the peak value in the crossspectrum, $\left[S_{p u}\right]_{\max }$, is plotted against the gap-todiameter ratio in Figure 10, where $p$ is the reference pressure and $u$ is the velocity at the representative point around the crossing as shown in Figure 7. The measurements were carried out twice, and the repeatability is excellent as seen in the Figure. Sharp maximum peaks appear in the plane of $\left[S_{p u}\right]_{\max }-s / d$ both in the trailing vortex region $(0 \leq s / d \leq 0.25)$ and in the necklace vortex region $(0.25 \leq s / d \leq 0.5)$, showing that these vortices shed most regularly at the gap-todiameter ratios $s / d=0.08$ and 0.28 , respectively. Figures 9 and 10 show that the periodicity of the vortex shedding is more regular for the necklace vortex than for the trailing vortex, and that the structure of flow changes abruptly at $s / d=0.25$ from trailing vortex region to necklace vortex region.

\section{3 Configurations of the longitudinal vortices}

To investigate the configurations of the longitudinal vortices, the two values of $s / d$ corresponding to the highest peaks of [ $\left.S_{p u}\right]_{\max }-s / d$ curve in Figure 10 were selected as representatives of the two regions, namely $s / d=0.08$ for the trailing vortex and $s / d=0.28$ for the necklace vortex. While the pressure tap for $p$ was fixed at the reference point on the upstream cylinder surface, the hot wire probe was scanned over a range near the crossing. From the cross-spectra between $p$ and $u$ obtained in this way, iso-value contour maps of

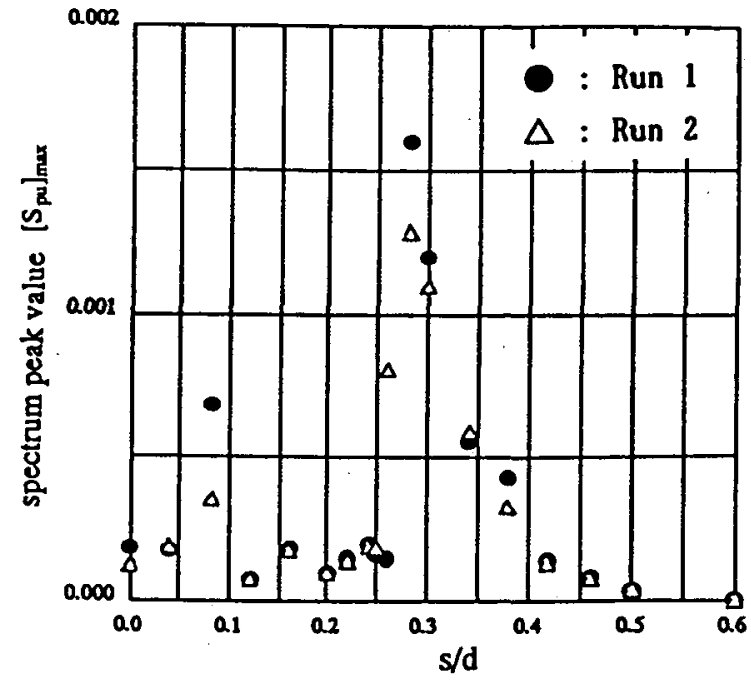

Fig. 10 Variation of the Height of the Peak in the Crossspectrum with the Gap-to Diameter Ratio. Experimental Conditions are identical with those for Figure 8.

$\left[S_{p u}\right]_{\max }$ were plotted as shown in Figures 11 and 12 for the two values of $s / d$. These maps yield information about the spatial structure of the longitudinal vortices. since they show the extent of the domain in the flow field where the velocity fluctuations include components synchronizing with the reference pressure on the upstream cylinder surface. From all the maps as shown in these figures, domains where the velocity is strongly influenced by the periodic shedding of the two types of longitudinal vortices are constructed and shown in Figure 13. It can be seen that the location and shape of areas affected by the trailing and the necklace vortices are definitely different from each other. It should be noted here that the shapes of the areas in Figure 13 do not express the. instantaneous configurations of the vortices. The configurations of the vortices change in a period of shedding and the areas in Figure 13 reflect the extent where the velocity $u$ is affected by the periodic shedding of the vortices.

The two longitudinal vortices were visualized in the water tunnel for their corresponding representative values of $s / d$. Their photographs are reproduced in Figures 14 and 15. By comparing these figures, definite difference can be discerned between the structures of the two vortices. In Figure 14, two trailing vortices are seen to form simultaneously on the same side of the upstream cylinder. A necklace vortex' around the downstream cylinder with a structure connected to $y$ $=0$ plane can be observed in Figure 15. The location and extent of these vortices correspond well with the corresponding counterparts in Figure 13. Based on the 


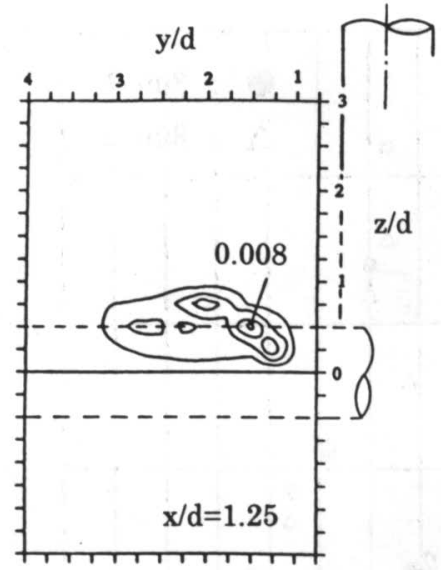

(a) on $y-z$ plane

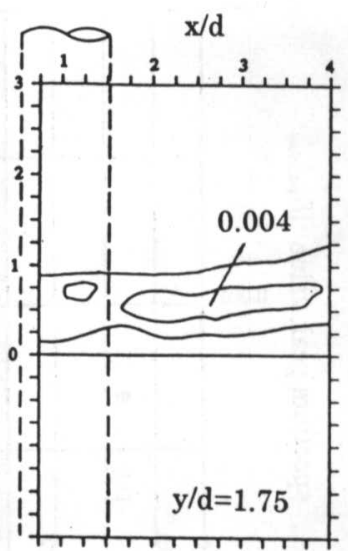

(b) on $x-z$ plane
Fig. 11 Iso-peak Contour Map for the Trailing Vortex obtained from Cross-spectra $(s / d=0.08)$.

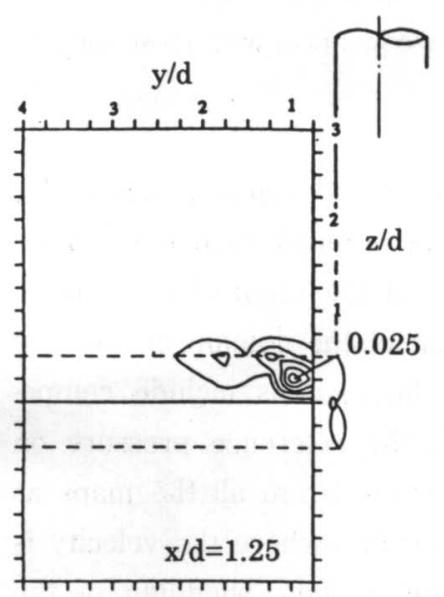

(a) on $y-z$ plane

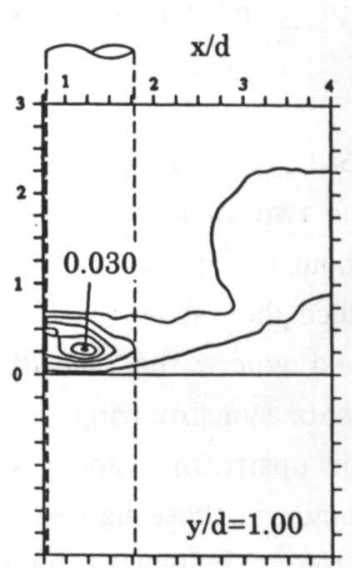

(b) on $x-z$ plane
Fig. 12 Iso-peak Contour Map for the Necklace Vortex obtained from Cross-spectra $(s / d=0.28)$.

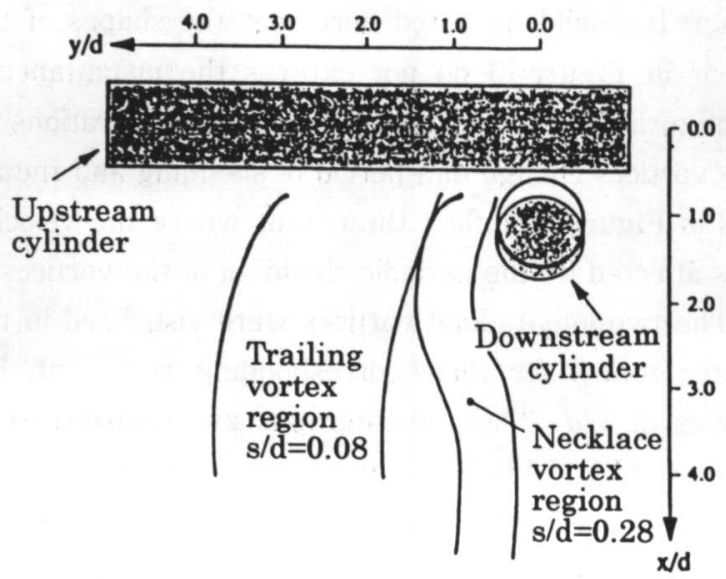

Fig. 13 Domains influenced by Periodic Shedding of the Longitudinal Vortices.

photographs as shown in Figures 14 and 15, together with direct observation of visualized flows with the aid of VTR, the structure of the two vortices at their most

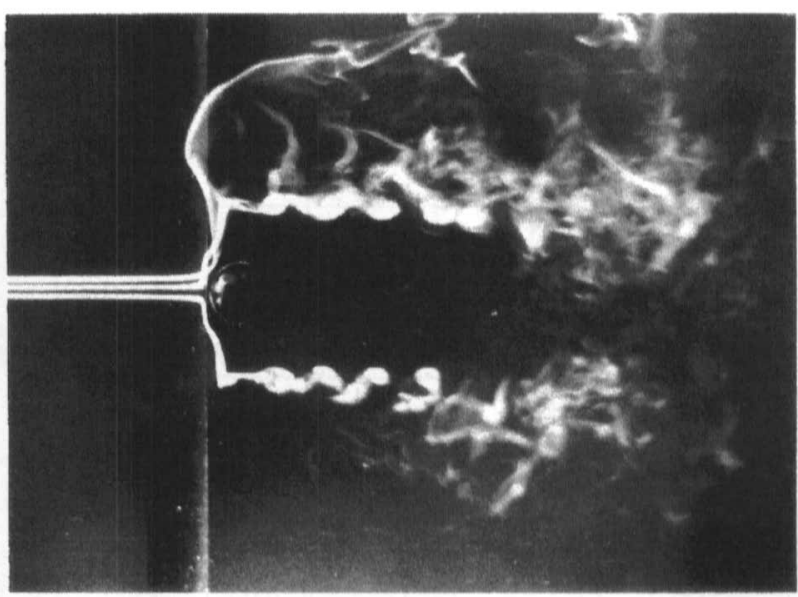

$\stackrel{\mathrm{y}}{\longrightarrow} \mathrm{x}$ (a) Top view (from $\mathrm{z}$ direction)

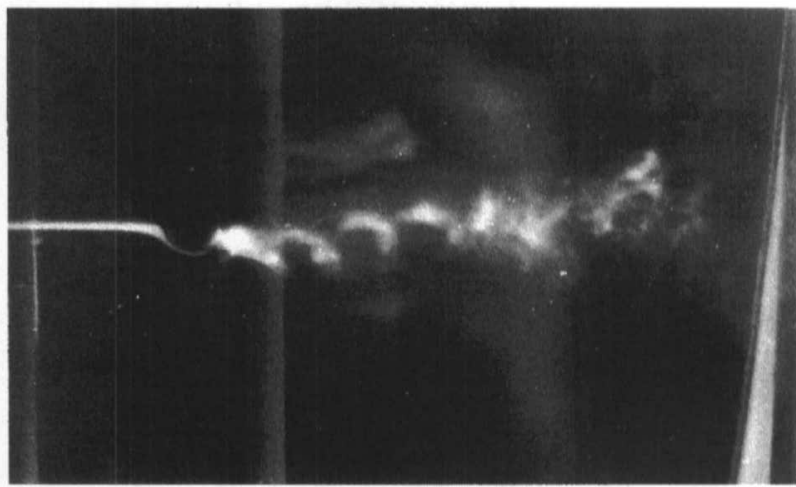

$\underset{\mathrm{b}_{\mathrm{z}}}{\longrightarrow} \mathrm{x} \quad$ (b) Side view (from $\mathrm{y}$ direction)

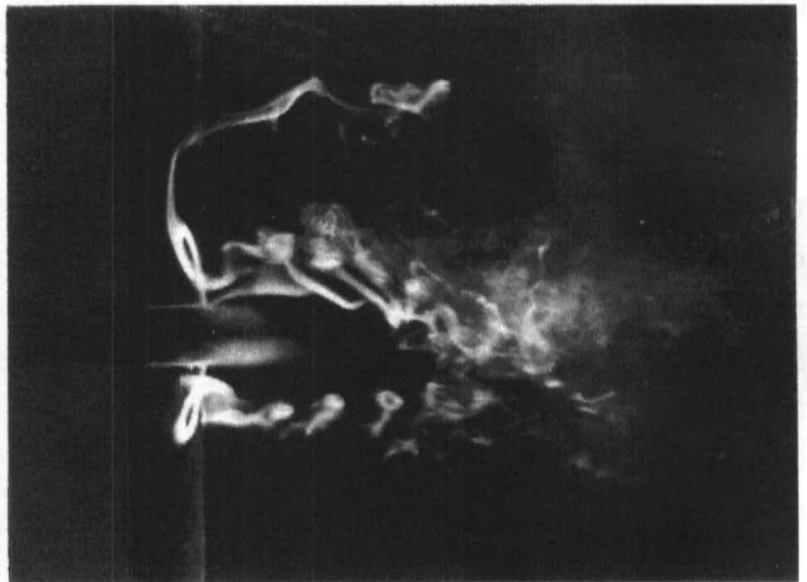

(c) Oblique view (from ' $\mathrm{A}$ ' direction in Fig.1)

Fig. 14 Photographs of Trailing Vortex visualized in the Water Tunnel $(d=10 \mathrm{~mm}, U=12.8 \mathrm{~cm} / \mathrm{s}, R e=$ $860)$.

developed stages are constructed as shown in Figure 16. 

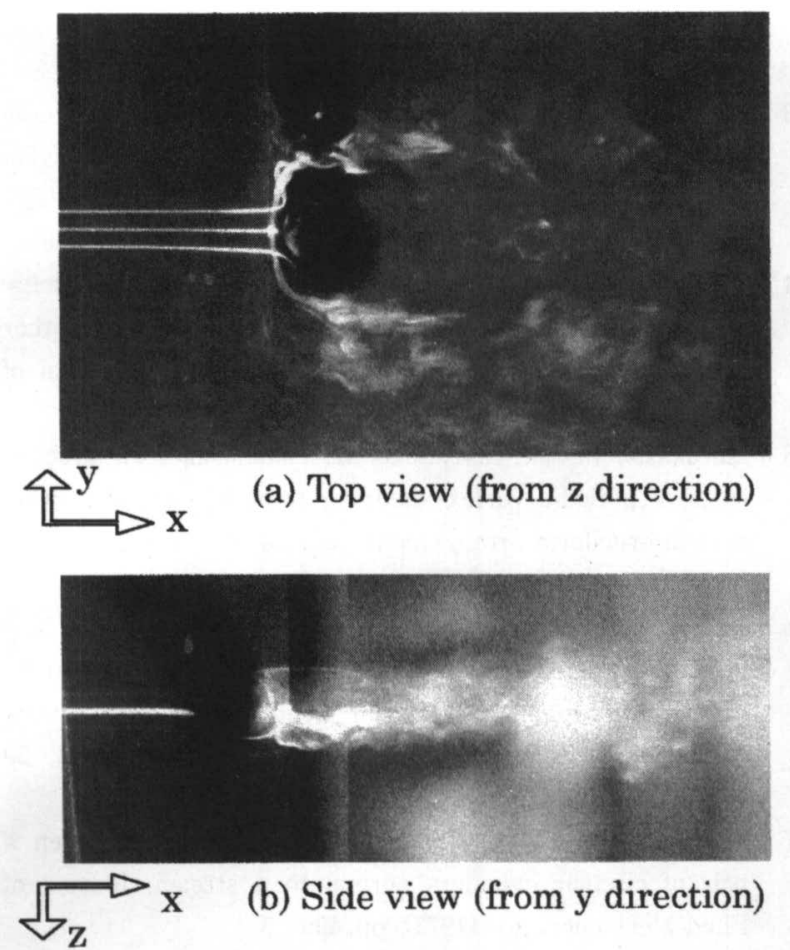

Fig. 15 Photographs of Necklace Vortex visualized in the Water Tunnel $(d=10 \mathrm{~mm}, U=12.5 \mathrm{~cm} / \mathrm{s}, R e=$ $900)$.
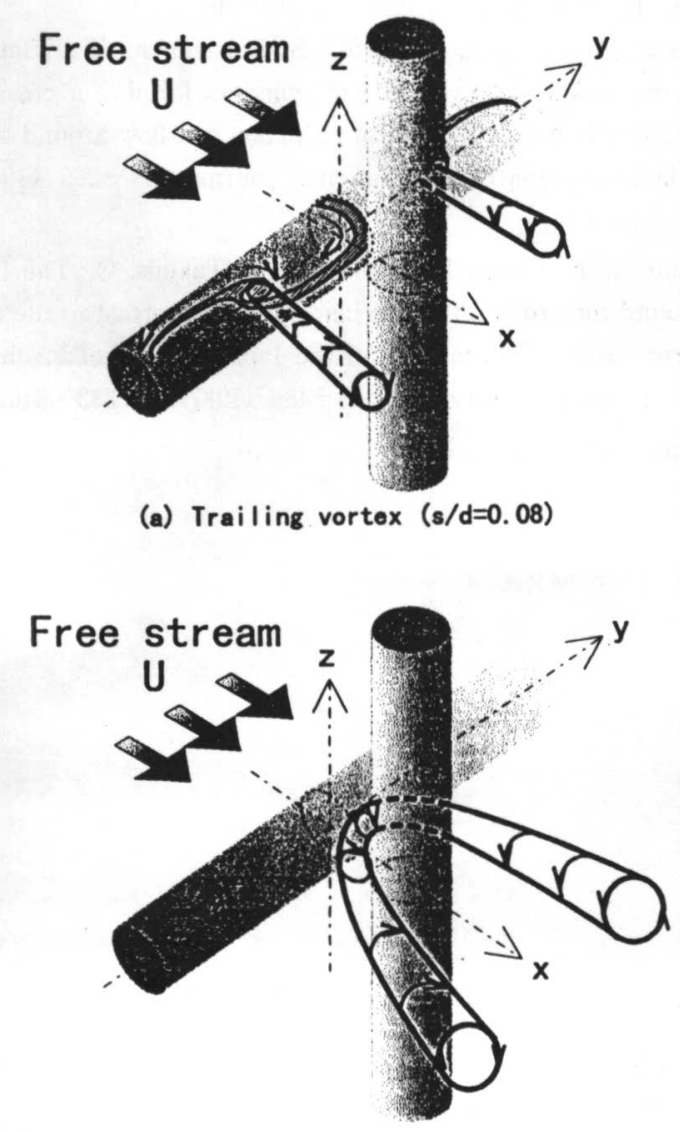

(b) Necklace vortex $(s / d=0.28)$

Fig. 16 Improved Vortex Models.
3.4 Dependence of Strouhal number on Reynolds number

The vortex shedding frequency $f_{v M}$ was counted directly in the visualized flow, or obtained from the spectrum of the velocity measured by hot film anemometry in the water tunnel experiment. In Figure 17 the Strouhal numbers for $f_{v M}$ obtained through water tunnel experiments are plotted against the Reynolds number $R e$ together with those obtained through wind tunnel experiments. Results obtained through the wind tunnel and the water tunnel correlate well and the dependence of $S t_{M}$ on $R e$ can clearly be seen in the Figure for $R e$ numbers lower than reported so far. The trailing vortex is formed steadily $\left(S t_{M}=0\right)$ when $R e<$ 1,000 , then increases steeply with increasing $R e$ and finally attains a constant value of around 0.09 at $R e=$ 10,000 . The necklace vortex was observed to shed periodically over the experimental range of $R e$, say $R e>$ 500. Its $S t_{M}$ gradually increases with $R e$ in the range of $R e<10,000$ and attains a practically constant of value around 0.04 when $R e>10,000$.

\section{Conclusions}

To investigate the origin of the new excitation of the upstream cylinder caused by the downstream counterpart, the flow structure around two fixed circular cylinders in cruciform arrangement placed in a uniform flow was investigated by using a wind tunnel and a water tunnel. Cross-spectra between the pressure at a reference point on the upstream cylinder surface and velocities around the crossing showed that the dominant frequency component in the latter synchronizes with that of in the former, verifying that the origin of the excitation lift force acting on the upstream cylinder is the same with that of the one causing periodic change in the velocity signal, i.e. the periodic shedding of either the trailing vortex or the necklace vortices.

Since the trailing vortex and the necklace vortex were found to shed most regularly when the gap-todiameter ratio is 0.08 and 0.28 , respectively, the configurations of the vortex shedding areas were investigated from the pressure-velocity cross-spectra for these values of $s / d$. The results obtained in this way corresponded well with the direct observation of the vortices visualized in the water tunnel. As a result models for the two longitudinal vortices are improved as shown in Figure 16, which are more definite and reliable in shape and size than those presented in earlier papers since the former are based on both direct observations and quantitative measurements. 


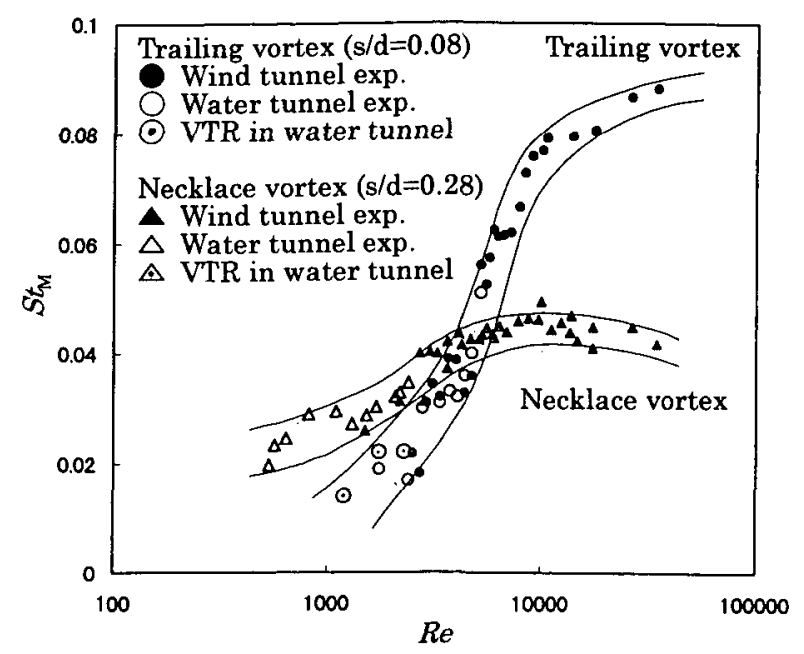

Fig. 17 Relationship between $S t_{M}$ and $R e$ (fixed system).

Experiments using wind and water tunnels clarified the dependence of the Strouhal number for the two longitudinal vortices over a wide range of $R e$. While the Strouhal numbers for the two vortices are virtually constant values when $R e>10,000$, as reported so far, they increase with appreciable gradients with increasing $R e$ when $R e<10,000$.

\section{Acknowledgement}

We thank Mr. K. Higashi, F. Hosaka and H. Tanaka for their great contribution in carrying out the experiments.

\section{References}

1) Bokaian, A., Geoola, F.: Wake-induced galloping of two interfering circular cylinders. Journal of Fluid Mechanics, 146 (1984) $383-415$.

2 ) Gowda, B.H.L., Prabhu, D.R. : Interference effects on the flow- induced vibration of a circular cylinder, Journal of Sound and Vibration, 112 (1987) 487-502.

3 ) Shirakashi, M., Yamagata, H. : Influence of a neighboring counterpart on the vortex induced oscillation of a circular cylinder, Transactions of the Japan Society of Mechanical Engineers, Series B, 53-495 (1987) pp. 3281-3284 (in Japanese).

4) Shirakashi, M., Mizuguchi, K., Bae, H.M. : Flow-induced excitation of an elastically. supported cylinder caused by another located downstream in cruciform arrangement, Journal of Fluids and Structures, 3 (1989) pp: 595-607.

5 ) Shirakashi, M., Bae, H.M., Sano, M., Takahashi, T.: Characteristics of periodic vortex shedding from two circular cylinders in cruciform arrangement, Journal of Fluids and Structures, 8 (1994) pp. $239-256$.

6) Bae. H.M., Takahashi, T., Shirakashi, M.: Suppression of Kármán vortex excitation of a circular cylinder by another located downstream in cruciform arrangement, Transactions of the Japan Society of Mechanical. Engineers, Series B, 59 -557 (1993) pp. 1-7, (in Japanese).

7) Bearman, P.W., Wadcock, A.J.: The interaction between a pair of circular cylinders normal to a stream, Journal of Fluid Mechanics, 61 (1973) pp. 499-511.

8 ) Fox, T.A.: Flow visualization at the center of a cross composed of tubes, International Journal of Heat and Flow, 11 (1990) pp. 160-162.

9) Fox, T.A.: Wake characteristics of two circular cylinders arranged perpendicularly to each other, ASME Journal of Fluids Engineering, 113 (1991) pp. 45-50.

10) Tomita, Y., Inagaki, S., Suzuki, S., Muramatsu. H. : Acoustic characteristics of two circular cylinders forming a cross in uniform flow (Effect on noise reduction and flow around both cylinders), JSME International Journal, 30-265 (1987) pp. $1069-1079$.

11) Yamada, H., Osaka, H; Kageyama, Y., Takeda, O.: The flow around the crossed two circular cylinders normal to the uniform stream. Transactions of the Japan Society of Mechanical Engineers, Series B, 53-486 (1987) pp. 333-340, (in Japanese). 